Departamento de Humanidades y Formación Integral Coordinación en procesos de Lectoescritura Oficina de Educación Virtual (UDCFD)

\title{
LOS AMBIENTES VIRTUALES DE APRENDIZAJE: HERRAMIENTAS DE APOYO PARA EL TRABAJO INDEPENDIENTE DEL ESTUDIANTE DE LA USTA
}

Clara Inés Jaramillo Gaviria Carlos Eduardo Álvarez Martínez 


\section{Departamento de Humanidades y Formación Integral Coordinación en procesos de Lectoescritura Oficina de Educación Virtual (UDCFD)}

Claramente se ve que es una necedad que los preceptores no hagan para ellos y los discípulos una tal distribución de los estudios que no solamente vayan unas cosas después de otras, sino que cada una de ellas se desenvuelva dentro de límites determinados. Sin determinar el límite ni fijar los medios para llegar a estos límites y el orden de estos medios, con facilidad se pasa algo, algo se invierte y se perturba todo. Así, pues:

1. El núcleo de los estudios debe distribuirse cuidadosamente en clases, a fin de que los primeros abran el camino a los posteriores y les den sus luces.

II. Hay que hacer una escrupulosa distribución del tiempo para que cada año, mes, día y hora tenga su particular ocupación.

III. Debe observarse estrictamente la extensión del tiempo y el trabajo para que nada se omita ni se trastorne nada. 


\section{Departamento de Humanidades y Formación Integral \\ Coordinación en procesos de Lectoescritura \\ Oficina de Educación Virtual (UDCFD)}

\section{NUESTRA PROPUESTA}

El propósito de esta ponencia es presentar los aspectos relevantes y problemáticos de la experiencia del uso de Ambientes Virtuales de Aprendizaje como herramienta de apoyo para las horas de trabajo independiente de los estudiantes en los cursos en la Educación Superior. La siguiente pregunta servirá de hilo conductor para esta reflexión: ¿Cómo promover el uso de los Ambientes Virtuales de Aprendizaje como herramientas de apoyo para el trabajo independiente de los estudiantes en los cursos de la modalidad presencial? Frente a esta pregunta, queremos defender la siguiente tesis:

La virtualidad no es opuesta a la modalidad presencial, pues el uso de Ambientes Virtuales de Aprendizaje, como herramientas de apoyo para el trabajo independiente, enriquece y complementa los procesos educativos presenciales; no obstante, las instituciones de Educación Superior y la comunidad académica (directivos, profesores y estudiantes) todavía no generan las condiciones de posibilidad para su óptimo desarrollo, ya que su énfasis principal sigue orientado hacia la modalidad presencial tradicional. 
Departamento de Humanidades y Formación Integral Coordinación en procesos de Lectoescritura Oficina de Educación Virtual (UDCFD)

\section{LOS CRÉDITOS ACADÉMICOS \\ Y LA DISTRIBUCIÓN DE HORAS PARA EL TRABAJO INDEPENDIENTE DEL ESTUDIANTE}

tiempo de trabajo dirigido

tiempo de trabajo independiente

El Crédito Académico equivale a 48 horas totales de trabajo del estudiante (MEN), horas académicas con acompañamiento docente

horas en actividades independientes de estudio, prácticas, preparación de exámenes

una hora de clase implica dos horas adicionales de trabajo independiente en pregrado y tres en posgrado (Ministerio de Educación Nacional, 2001).

En la modalidad presencial se le hace poco seguimiento al proceso autónomo del estudiante.

AVA guía en el trabajo independiente de los estudiantes

Profundización ----- dificultad teórica, práctica

refuerzo

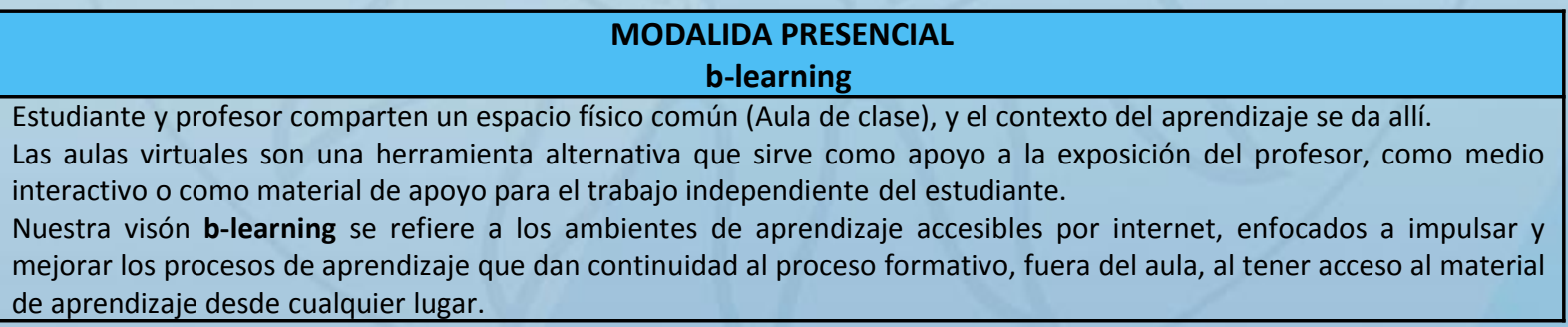


Departamento de Humanidades y Formación Integral

Coordinación en procesos de Lectoescritura

Oficina de Educación Virtual (UDCFD)

\section{PROPUESTA DEL USO DE LOS AVA EN LA MODALIDAD PRESENCIAL}

Explicación del tema

Modelación del docente

Aplicación del ejercicio en

clase
AVA: Temas específicos trabajados en clase (Grado de dificultad en comprensión y apropiación)
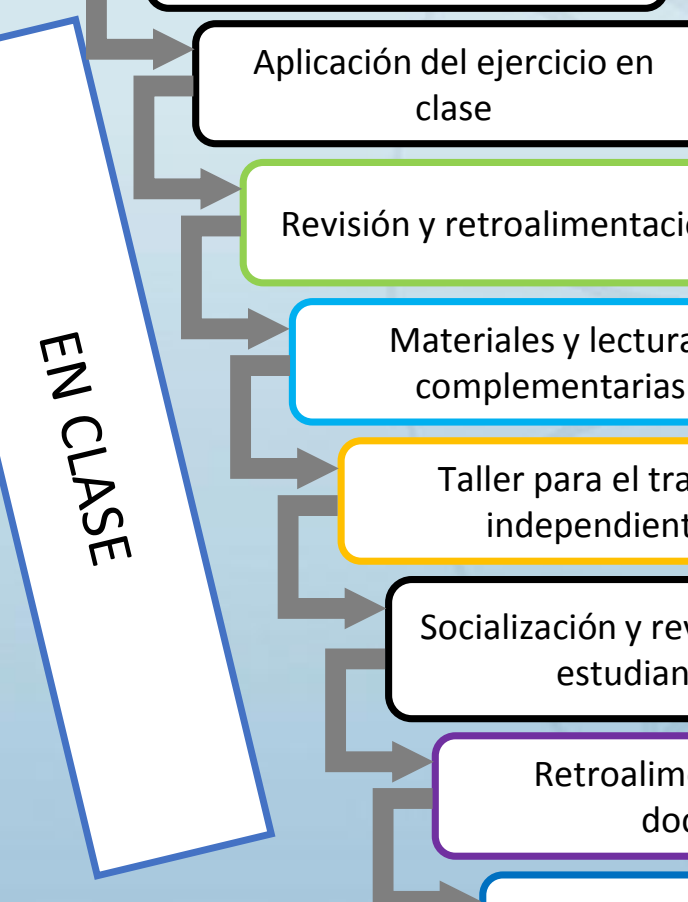

Revisión y retroalimentación

Materiales y lecturas complementarias

Taller para el trabajo independiente

Socialización y revisión de los estudiantes

Retroalimentación del docente

OVA: Material para el estudio modelación - ejercicios de aplicación

Materiales y lecturas complementarias

Ejercicios de apoyo

Ejercicios para evaluar lo aprendido

Socialización y revisión de los estudiantes

$$
\text { clase }
$$




\section{EL AVA COMO HERRAMIENTA DE APOYO PARA LA CLASE PRESENCIAL}

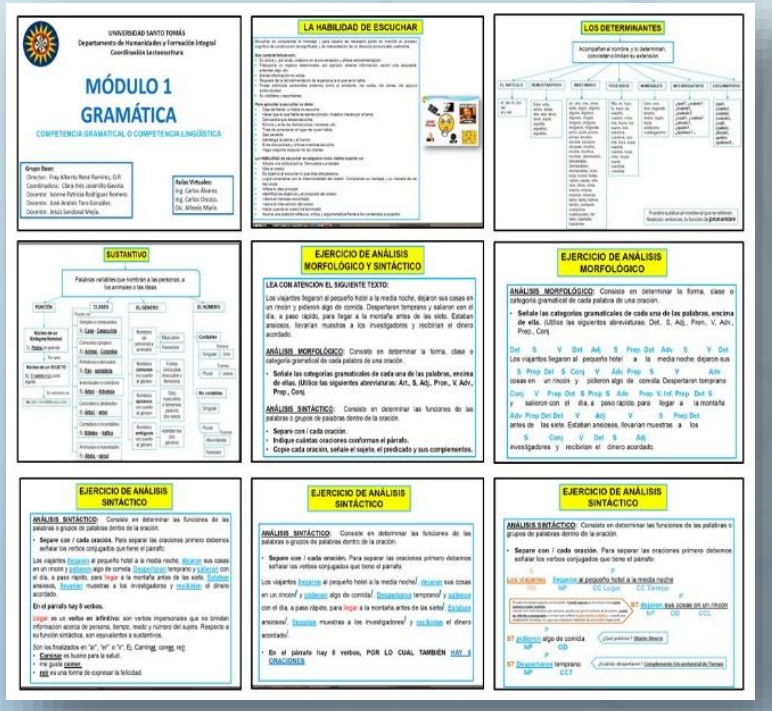

$\checkmark$ Se lee el material de fundamentación que se va a trabajar en clase;

$\checkmark$ Se realiza un ejercicio de modelación del análisis morfológico y sintáctico de un párrafo;

$\checkmark$ Se solucionan las dudas e interrogantes que surgen con respecto al tema tratado;

$\checkmark$ Se proponen ejercicios de dificultad considerable que se desarrollan en compañía del docente con el fin que los estudiantes sigan paso a paso el ejemplo elaborado e interioricen el conocimiento, para que finalmente puedan ser evaluados mediante un taller de clase. 
Departamento de Humanidades y Formación Integral Coordinación en procesos de Lectoescritura Oficina de Educación Virtual (UDCFD)

\section{EL AVA COMO HERRAMIENTA DE APOYO PARA LAS HORAS DE TRABAJO INDEPENDIENTE DEL ESTUDIANTE}

En las horas de trabajo independiente, el estudiante, que en clase identificó los temas que debe repasar o reforzar, debe:

$\checkmark$ ingresar al aula virtual estudiar en el material para el aprendizaje (OVA),

$\checkmark$ desarrollar los ejercicios prácticos para que en la medida que va adquiriendo los conceptos los aplique en contexto;

$\checkmark$ complementar o profundizar lo aprendido con materiales de apoyo disponible en el AVA. En este caso encuentra un recurso que le posibilita repasar algunas reglas ortográficas.

$\checkmark$ Realizar ejercicios de refuerzo con el fin que autoevalúe y determinar si requiere volver a estudiar algún tema $\in \mathrm{n}$ específico.

- Socializar, en clase presencial, el trabajo realizado durante las horas de Trabajo independiente para despejar dudas.

- Evaluar el proceso, se retroalimenta y se califica.
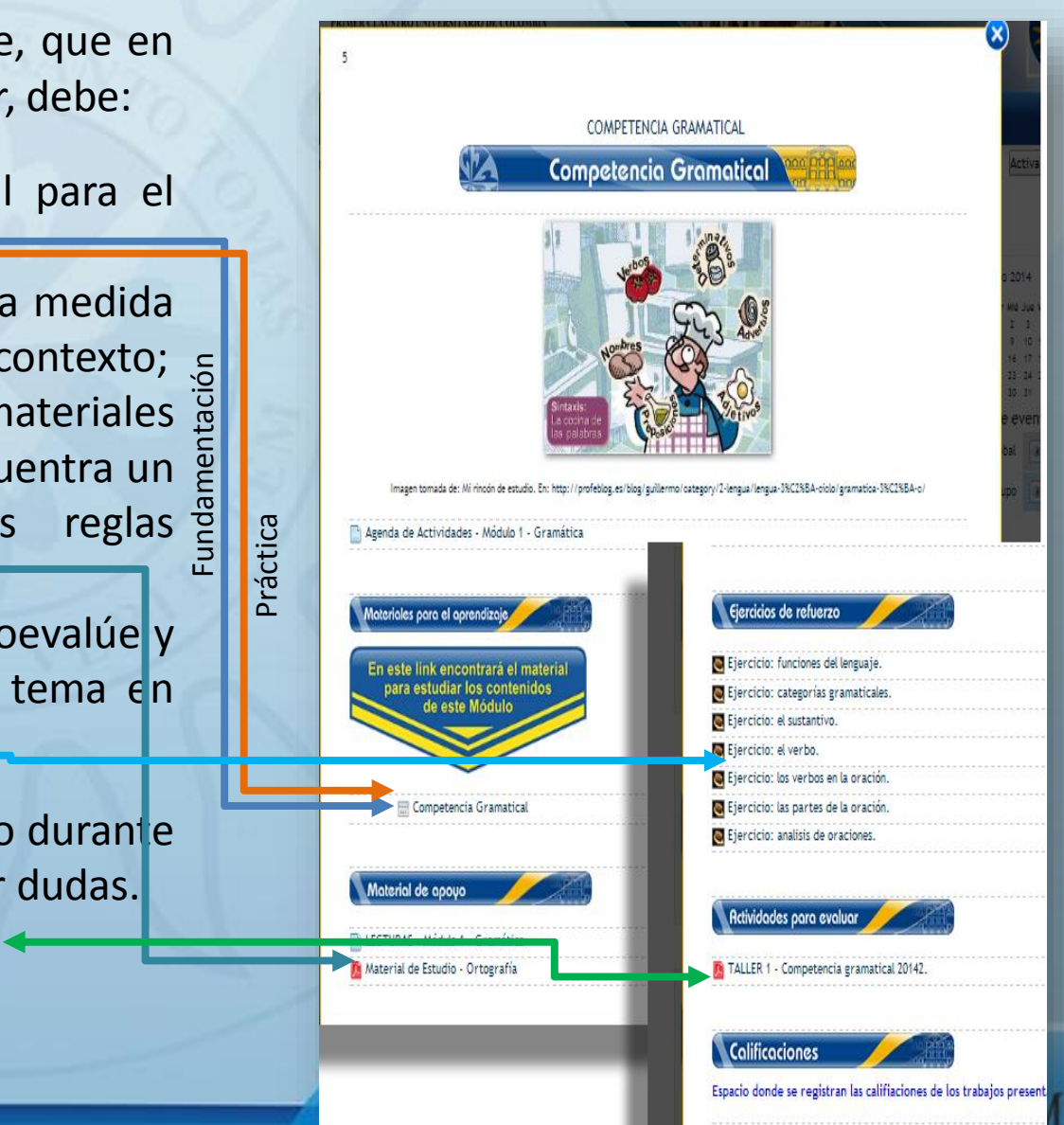
Departamento de Humanidades y Formación Integral Coordinación en procesos de Lectoescritura Oficina de Educación Virtual (UDCFD)

\section{CARACTERIZACIÓN DE LOS CURSOS IMPLEMENTADOS EN LA OFERTA FORMATIVA DE ACOMPAÑAMIENTO EN LECTOESCRITURA}

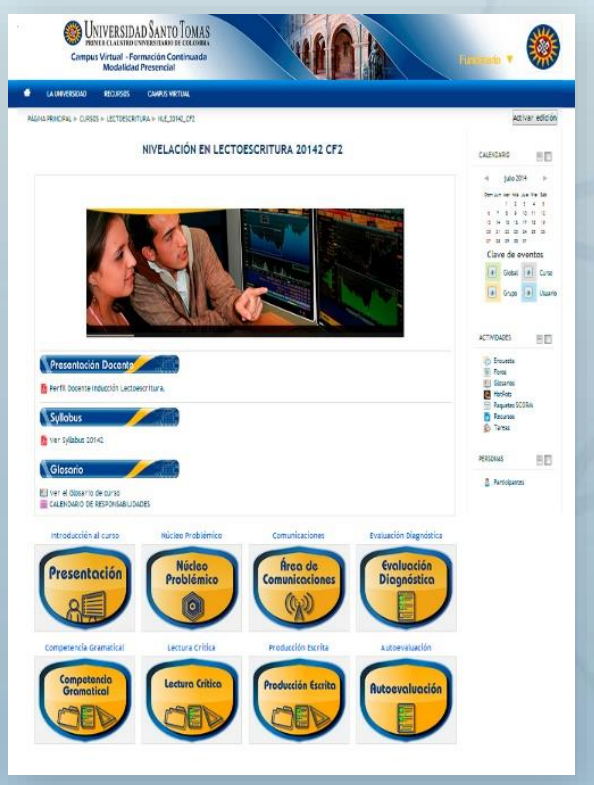

\begin{tabular}{|c|c|c|c|}
\hline Periodo & AVA creados & $\begin{array}{c}\text { Estudiantes } \\
\text { inscritos }\end{array}$ & $\begin{array}{l}\text { Estudiantes } \\
\text { que utilizan } \\
\text { el AVA }\end{array}$ \\
\hline 2013-2 & $\begin{array}{l}\text { - } 18 \text { aulas para presencial. } \\
\text { - } 1 \text { aula para nivelación virtual. } \\
\text { - } 1 \text { aula para el Diplomado para docentes. }\end{array}$ & 507 & 303 \\
\hline 2014-1 & $\begin{array}{l}\text { - } 18 \text { aulas para presencial. } \\
\text { - } 1 \text { aula para nivelación virtual. } \\
\text { - } 2 \text { aula para el Diplomado para docentes. }\end{array}$ & 907 & 317 \\
\hline 2014-2 & $\begin{array}{l}\text { - } 18 \text { aulas para presencial. } \\
\text { - } 1 \text { aula para nivelación virtual. } \\
\text { - } 1 \text { aula para el Diplomado para docentes. }\end{array}$ & 475 & 175 \\
\hline $2015-1$ & $\begin{array}{l}\text { - } 18 \text { aulas para presencial. } \\
\text { - } 1 \text { aula para nivelación virtual. } \\
\text { - } 1 \text { aula para el Diplomado para docentes. }\end{array}$ & & \\
\hline
\end{tabular}


Departamento de Humanidades y Formación Integral Coordinación en procesos de Lectoescritura Oficina de Educación Virtual (UDCFD)

\section{SEGUIMIENTO Y ACOMPAÑAMIENTO DEL ESPACIO VIRTUAL EN LOS CURSOS DE LECTOESCRITURA}

Estrategia de acompañamiento

- Uso efectivo de materiales, ejercicios y actividades

- Orientación docente

\begin{tabular}{|c|c|c|c|c|c|c|}
\hline $\begin{array}{l}\text { Periodo } \\
\text { Académic }\end{array}$ & Nombre del Curso & $\begin{array}{l}\text { Accesos Agenda } \\
\text { Módulo } 1\end{array}$ & $\begin{array}{c}\text { Accesos Recursos } \\
\text { SCORM }\end{array}$ & $\begin{array}{l}\text { Accesos Lecturas } \\
\text { Módulo } 1\end{array}$ & $\begin{array}{c}\text { Accesos Actividades } \\
\text { de Refuerzo }\end{array}$ & $\begin{array}{c}\text { Accesos Actividades } \\
\text { para Evaluar }\end{array}$ \\
\hline$\exists 20132$ & NIVELACIONN LECTO-ESCRITURA CFA & & & & 114 & 131 \\
\hline & NIVELACIONN LECTO-ESCRITURACFB & 20 & & 7 & & \\
\hline & NIVELACION LECTO-ESCRITURACSA & 80 & & 73 & 175 & 246 \\
\hline & NIVELACIOON LECTO-ESCRITURA CSB & 25 & & 10 & 33 & \\
\hline & NIVELACION LECTO-ESCRITURA DGA & 36 & & 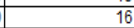 & 193 & 77 \\
\hline & NIVELACION LECTO-ESCRITURADGB & 30 & & 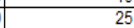 & 67 & 101 \\
\hline & NIVELACION LECTO-ESCRITURADRA & 67 & & 25 & 38 & \\
\hline & NIVELACION LECTO-ESCRITURADRB & 62 & & 31 & 59 & \\
\hline & NIVELACION LECTO-ESCRITURADRC & 19 & & 18 & 50 & \\
\hline & NIVELACIOON LECTO-ESCRITURA DRD & 55 & & 34 & 101 & \\
\hline & NIVELACIÓN LECTO-ESCRII & 42 & & 21 & 73 & \\
\hline & NIVELACIÓN LECTO-ESCRI & 16 & & 19 & 16 & \\
\hline & NIVELACION LECTO-ESCRIT & 30 & & 24 & 30 & \\
\hline & VLECTO-ESCRITURA PSA & 40 & & 2 & 42 & 152 \\
\hline & ION LECTO-ESCRIT & 55 & & 46 & 256 & \\
\hline & NIVELACIÓN LECTO-ESCRITURAREP & 4 & & 2 & 65 & \\
\hline & NIVELACIÖN LECTO-ESCRITURAREP1 & 1 & & & 0 & \\
\hline & NIVELACIÓN LECTO-ESCRITURA SOC & 23 & & & 120 & 34 \\
\hline$\exists 20141$ & NIVELACIÓN EN LECTOESCRITURA 20141 CFA & & & & & \\
\hline
\end{tabular}

Fuentes de seguimiento

- Informe de actividad

- Bases de datos

(SELECT COUNT(1.id) AS Registros FROM prefix_log AS 1

WHERE 1. user id $=u$. id AND 1. course $=$ c. id AND 1. $\mathrm{cmid}=$ (SELECT id FROM prefix_course_modules AS CmO WHERE cmo. course $=$ c. id AND

- Consolidado de accesos por tipo de cmo.module =(SELECT id FROM prefix_modules AS mO WHERE

contenido en el módulo: agenda,

mo. name $=$ 'scorm') AND cmo. instance $=$ (SELECT id FROM prefix_scorm AS recursos SCORM, lecturas del modulo. cour $s e=c$. id))

módulo, actividades de refuerzo y ) actividades para evaluar.

As competenciagramatical

- Consolidado de acceso por módulo 
Departamento de Humanidades y Formación Integral Coordinación en procesos de Lectoescritura Oficina de Educación Virtual (UDCFD)

\section{SEGUIMIENTO Y ACOMPAÑAMIENTO DEL ESPACIO VIRTUAL EN LOS CURSOS DE LECTOESCRITURA}

A medida que avanza el curso de lectoescritura, los estudiantes se apropian de las temáticas tratadas en clase y complementadas en los materiales de refuerzo y los ejercicios prácticos y de evaluación. Esto hace que en los siguientes temas a desarrollar requieren menor apoyo y por ende se da la disminución en la consulta de recurso educativos y actividades de formación.

\begin{tabular}{|r|l|r|r|r|}
\hline $\begin{array}{l}\text { Periodo } \\
\text { Académico }\end{array}$ & Nombre del Curso & $\begin{array}{l}\text { Módulo 1. } \\
\text { Competencia } \\
\text { Gramatical }\end{array}$ & $\begin{array}{l}\text { Módulo 2. } \\
\text { Lectura } \\
\text { Crítica }\end{array}$ & $\begin{array}{l}\text { Módulo 3. } \\
\text { Producción } \\
\text { Escrita }\end{array}$ \\
\hline 20132 & NIVELACIÓN PRESENCIAL & $50 \%$ & $30 \%$ & $20 \%$ \\
\hline 20141 & NIVELACIÓN PRESENCIAL & $15 \%$ & $12 \%$ & $4 \%$ \\
\hline 20142 & NIVELACIÓN PRESENCIAL & $47 \%$ & $39 \%$ & $14 \%$ \\
\hline Total general & & $36 \%$ & $25 \%$ & $12 \%$ \\
\hline
\end{tabular}




\section{Departamento de Humanidades y Formación Integral \\ Coordinación en procesos de Lectoescritura \\ Oficina de Educación Virtual (UDCFD)}

\section{CONCLUSIÓN}

A manera de síntesis, podemos afirmar que:

- Los AVA son un recurso al servicio del proceso de enseñanza y aprendizaje en la modalidad presencial de la Educación Superior.

- Su optimización requiere un cambio de paradigma organizacional, administrativo y tecnológico, que permita crear equipos de trabajo colaborativo articulados con las perspectivas académicas y pedagógicas expuestas en el PEl, el Modelo Pedagógico, el perfil de los egresados y los lineamientos curriculares de cada Institución, con el objetivo de dar sentido al uso de los AVA en el proceso de enseñanza y aprendizaje y motive a la innovación de herramientas didácticas que respondan a los retos que propone la sociedad del conocimiento.

Tras esta breve exposición y fundamentados en la experiencia que se ha proporcionado desde los cursos de lectoescritura en la USTA, podemos ratificar la idea de que la modalidad virtual no es opuesta a la presencial, pues el uso de ambientes virtuales de aprendizaje, como herramientas de apoyo para el trabajo independiente, enriquece y complementa los procesos educativos presenciales; no obstante, la comunidad académica y las instituciones de educación superior deben propender por promover las condiciones de posibilidad para su óptimo desarrollo, ya que su énfasis principal sigue orientado hacia la modalidad presencial tradicional. 


\section{CONCLUSIÓN}

En la actualidad, algunas Instituciones de Educación Superior no cuentan con los recursos tecnológicos adecuados para garantizar una conectividad eficiente, se trabaja con la limitación de software libre pues es poca la inversión que se hace en la adquisición de programas especializados, las salas de sistema son deficientes para la demanda de sus usuarios, etc. A este gran problema subyacen otras dificultades como las que enuncian Andrés Felipe Velásquez y Eduardo Augusto López Ramírez en su investigación, "Una mirada crítica al papel de las TIC en la educación superior en Colombia":

Entre los principales problemas que se presentan actualmente en la educación superior mediada por entornos virtuales de aprendizaje se encuentran:

1. Ausencia de estrategias didácticas en el diseño de material docente específico para uso en entornos virtuales de aprendizaje (Tradicionalmente los materiales son simples documentos elaborados en Word, Excel y Power Point diseñados para la educación presencial).

2. Bajo nivel de formación docente en el dominio de competencias sobre entornos virtuales de aprendizaje.

3. Falta de políticas y estrategias institucionales sobre la implementación y uso adecuado de los entornos virtuales de aprendizaje (Velásquez \& López Ramírez, 2010). 


\section{Departamento de Humanidades y Formación Integral \\ Coordinación en procesos de Lectoescritura \\ Oficina de Educación Virtual (UDCFD)}

\section{BIBLIOGRAFÍA}

Cabero, J., \& Román, P. (2011). E-Actividades. Un referente básico para la formación en Internet. Sevilla: Aula Múltiple - Magisterio.

Gavils Panqueva, Á. (1998). Ambientes virtuales para participación en I sociedad del conocimiento. (UNIANDESLIDIE, Ed.) Informática Educativa, 11(2), 247-260.

Ministerio de Educación Nacional (MEN). (15 de marzo de 2012). Al día con las noticias. Monitoreo de prensa. Obtenido de Universitarios están mal en inglés y en comprensión de lectura: http://www.mineducacion.gov.co/observatorio/1722/article-300079.html

Ministerio de Educación Nacional. (10 de noviembre de 2001). Mineducación. Obtenido de Altablero: http://www.mineducacion.gov.co/1621/article-87727.html

Sunkel, G. (8 de julio de 2011). Red Latinoamericana Portal educativo. Obtenido de TIC para la educación en América Latina: hacia una perspectiva integral: http://www.relpe.org/especial-del-mes/tic-para-la-educacion-enamerica-latina-hacia-una-perspectiva-integral/

UNESCO. (9 de octubre de 1998). UNESCO Educación. Obtenido de Declaración mundial sobre la educación superior en el siglo XXI: visión y acción y marco de acción prioritaria para el cambio y el desarrollo de la educación superior: http://www.unesco.org/education/educprog/wche/declaration_spa.htm

Velásquez , A. F., \& López Ramírez, E. A. (2010). Una mirada crítica al papel de las TIC en la educación superior. Obtenido de E-Mail Educativo, 1(1): http://www.revistas.unal.edu.co/index.php/email/article/view/12623 


\section{潧 UNIVERSIDAD SANTOTOMAS

Departamento de Humanidades y Formación Integral Coordinación en procesos de Lectoescritura Oficina de Educación Virtual (UDCFD)

\section{GRACIAS POR SU ATENCIÓN Y APORTES}

